Tobias Salesch*, Jonas Gesenhues, and Dirk Abel

\title{
A Hybrid Mock Circulation for Control Algorithms of Ventricular Assist Devices
}

https://doi.org/10.1515/cdbme-2019-0103

\begin{abstract}
This paper deals with the design, simulation and control of a new lightweight hybrid Mock-Loop (MCL) concept. The proof of concept is evaluated by two simulation approaches. First, the design parameters are chosen by an optimal control problem. Second, a cascading controller structure is evaluated in a simulation. Both show that with a suitable range of the design parameter the new lightweight concept can be used as a MCL. To validate these findings, further investigations with the MCL under realistic test conditions are required.
\end{abstract}

Keywords: Mock Circulation, Automatic Control, Ventricular Assist Devices, Optimal Control

\section{Introduction}

The Institute of Automatic Control at RWTH Aachen University develops new control strategies for Left Ventricular Assist Devices (LVAD) to improve the therapy for patients with suffering endstage heart failure. Before these algorithms can be deployed in-vivo it is desirable to examine them in-vitro. For this purpose, a lightweight hybrid Mock-Loop (MCL) is designed. The concept aims to support the development process and evaluation of LVAD control strategies. Hardware-in-theloop (HIL) concepts of MCLs use existing numerical models of the cardiovascular system to calculate flow rates and pressures for different scenarios [1]. HIL concepts are already state of the art in the development process of LVADs and vary in use cases and design depending on their purpose ([1],[2], [3], [4], [5], [6], [7]). The newly developed concept is a combination of several existing approaches. According to [2], hybrid pressure chambers filled with fluid and air are used. Instead of a pneumatic system, gear pumps are used to apply desired pressures (and the amount of air is constant). Existing concepts use gear pumps regulating fluid flows to apply certain pressures but for systems without air [6]. Other approaches use the gear pumps only for circulation flow while high dynamic coils apply a pressure on the fluid [7]. Proportional pneumatic valves like they are used in [2] have hysteresis and a high pressure

*Corresponding author: Tobias Salesch, Institute of Automatic Control, Campus-Boulevard 30, RWTH Aachen University, Germany, e-mail: t.salesch@irt.rwth-aachen.de Jonas Gesenhues, Dirk Abel, Institute of Automatic Control, RWTH Aachen University, Germany

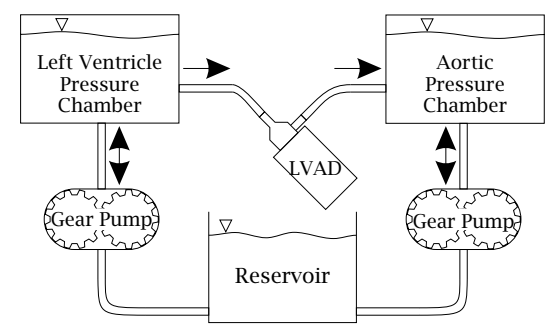

Fig. 1: An overview of the presented and investigated MCL design. A LVAD is connected to two pressure chambers, representing the left ventricular and aortic pressure. The pressures are applied by gear pumps. A reservoir delivers the required amount of fluid.

depended transmission behavior. Dynamic coils are expensive and difficult to drive. Accordingly, in the presented concept gear pumps are used due to their near proportional voltage to flow transmission behavior. Combining gear pumps with a hybrid fluid and air chamber promises advantages in controlling and simplification in electronics. Figure 1 shows the design in general. The LVAD is connected to two chambers representing the left ventricle and aortic pressure. Both hold a constant amount of air and for each a gear pump is used to change the fluid level and thus the pressure. A reservoir delivers the required amount of fluid.

The aims of this paper can be split into two separate parts. First suitable ranges for the gear pumps dynamics and the constant amount of air in the pressure chambers are examined in an optimal control problem. Second, a developed control approach is evaluated for the resulting system parameters of the optimal control problem. For both simulation studies upscaled in-vivo data of the left ventricle are used as reference. As the left ventricular pressure is dynamically more challenging compared to the aortic pressure, in this paper only the left ventricle is considered.

\section{Simulation Model}

In this section, the fundamental equations for the pressure cylinder and the gear pumps are presented. Those are used in the optimal control problem and control simulation.

The constant amount of air $n$ is modeled as ideal gas with the pressure $p$, the volume $V$, the temperature $T$, and the uni- 
versal gas constant $R$ :

$$
p \cdot V_{\text {air }}=n \cdot R \cdot T
$$

Air leakage as well as temperature changes of the air are neglected. Thus, the amount of air in the cylinder, the temperature and the ideal gas constant are defined as constants. Time derivating equation 1 will lead to:

$$
\dot{p}=-\frac{p \cdot \dot{V}_{\text {air }}}{V_{\text {air }}}
$$

The fluid is assumed to behave incompressible. Therefore the change of air volume $\dot{V}_{a i r}$ is expressed as LVAD and gear pump flow:

$$
\dot{V}_{\text {air }}^{L V}=\dot{V}_{\text {fluid }}^{V A D}-\dot{V}_{\text {fluid }}^{L V}
$$

The gear pumps flow for the left ventricle pressure cylinder $\dot{V}_{\text {fluid }}^{L V}$ is defined as positive when pumping fluid from the reservoir to the cylinder. The LVAD generates a flow $\left(\dot{V}_{\text {fluid }}^{V A D}\right)$ from the left ventricular to the aortic pressure chamber. Combining equation 2 and the integration of equation 3 leads to the following model for ventricular pressure variation $\dot{p}^{L V}$ with the unstressed air volume $V_{0}^{L V}$ :

$$
\dot{p}^{L V}=\frac{p^{L V} \cdot\left(\dot{V}_{\text {fluid }}^{L V}-\dot{V}_{\text {fluid }}^{V A D}\right)}{V_{\text {fluid }}^{V A D}-V_{\text {fluid }}^{L V}+V_{0}^{L V}}
$$

The gear pumps are driven by a standard DC motor without speed control. As the fluid is considered incompressible, every turn of the motor moves the same amount of fluid. Thus, the flow rate is proportional to the rotational speed. The voltage to angular velocity transmission behaviour of the DC motor is approximated as first order system with the time constant proportional to the overall moment of intertia of motor and gears [7] [8]. Thus, the differential equation of the gear pump without pressure influence is evolved:

$$
\ddot{V}_{\text {fluid }}^{\Delta p=0} \cdot T+\dot{V}_{\text {fluid }}^{\Delta p=0}=K \cdot u
$$

It depends on the input voltage $u$, a voltage to flow constant $K$ and the yet unknown time constant $T$ of the gear pump. A higher pressure on one side of the pump generates a moment against or in direction of rotation. Thus, the angular velocity changes and the flow is reduced or increased, respectively. The impact of the pressure difference between the inlet and outlet pressure $\Delta p=p_{L V}-p_{a t m}$ on the gear pumps flow $\dot{V}_{\text {fluid }}^{\Delta p}$ is considered as a constant proportional factor $k_{p}$ :

$$
\dot{V}_{\text {fluid }}^{\Delta p}=\dot{V}_{\text {fluid }}^{\Delta p=0} \cdot\left(1-\operatorname{sign}\left(\dot{V}_{\text {fluid }}\right) \cdot \Delta p \cdot k_{p}\right)
$$

Time derivating and rearranging equation 6 leads to:

$$
\begin{aligned}
a & =\left(1-\operatorname{sign}\left(\dot{V}_{\text {fluid }}\right) \cdot \Delta p \cdot k_{p}\right) \\
\dot{V}_{\text {fluid }}^{\Delta p=0} & =\frac{\dot{V}_{\text {fluid }}^{\Delta p}}{a} \\
\ddot{V}_{\text {fluid }}^{\Delta p=0} & =\frac{\ddot{V}_{\text {fluid }}^{\Delta p}}{a}+\operatorname{sign}\left(\dot{V}_{\text {fluid }}\right) \frac{\dot{V}_{\text {fluid }}^{\Delta p} \cdot \Delta \dot{p} \cdot k_{p}}{a^{2}}
\end{aligned}
$$

As the atmospheric pressure $p_{a t m}$ is constant, the derivative of $\Delta p$ is equal to the derivative of the pressures in the cylinder: $\Delta \dot{p}=\dot{p}_{L V}$. For the final transmission model of the gear pumps, equation 8 and equation 9 are put into equation 5 .

In this paper the dynamic pump constants of a MARCO UP2-P pump are investigated. Therefore, the pump was excited with voltage steps of $6 \mathrm{~V}, 12 \mathrm{~V}, 18 \mathrm{~V}$ and $24 \mathrm{~V}$ whereas the maximum voltage is limited to $24 \mathrm{~V}$. The resulting flows were analysed with Mathworks Matlabs System Identification Toolbox. The toolbox fitted the constants $K$ and $T$ to the first order lag element (equation 5). The pressure constant $k_{p}$ is picked from the pumps datasheet [10]. This results in an identified gear pump with $K=0.3583 \mathrm{~L} \mathrm{~min}^{-1} \mathrm{~V}^{-1}, T=0.1538 \mathrm{~s}$ and $k_{p}=2.3 \mathrm{~L} \mathrm{~min}^{-1} \mathrm{bar}^{-1}$.

The following simulation studies will only refer to this identified gear pump. Instead of randomly changing the gear pumps parameters, the amount of parallel UP2-P pumps for one pressure cylinder is increased. With more parallel pumps the dynamic is increased.

\section{Optimal Control Problem}

The aim of an optimal control problem is to identify the optimal input trajectory (over a defined time horizon) for a certain desired output reference trajectory. In this work the input corresponds to the voltage exciting the gear pumps and the output is the pressure in the left ventricular pressure chamber. The optimization goal is to follow the pressure reference as effectively as possible and thus, deviations from the reference are penalized quadratic in the optimization cost function. The problem is constrained as the maximum voltage applied to the gear pumps should not exceed an upper boundary defined by the manufacturer.

The problem is set up in Mathworks Matlab by using CasADI [9]. The non-linear programming problem is created by using a direct multiple shooting approach. The integration is done by using a forth order runge kutta procedure. The resulting non-linear initial value problems are solved using the IPOPT interior point method [12]. The problem is solved for wide range of the constant amount of air and one up to four parallel identified pumps to find a suitable range for both parameters.

For the controller the LVAD flow acts as a disturbance. Typically, a cardiovascular model is used to generate closedloop reference trajectories for the MCL pressures. As the aim of this work is to proof the newly developed concept, in-vivo trajectories from animal trials are used. The LVAD is driven at constant speed and the complete hardware set up is described in [11]. The recorded trajectories are upscaled to represent a 

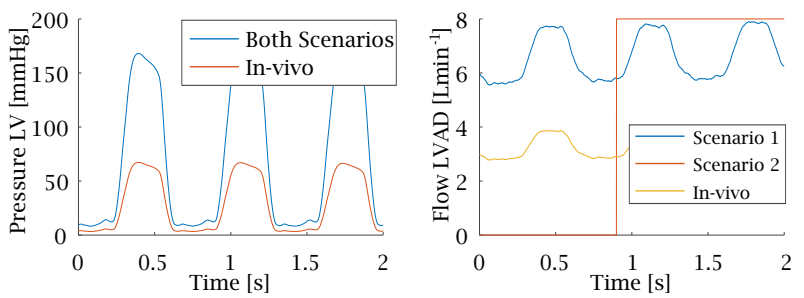

Fig. 2: On the left the reference and in-vivo pressure is shown. The right side points out the reference and in-vivo LVAD flow for both scenarios of the optimal control problem.

worst case scenario. A two second section of the in-vivo trajectories was chosen to be the reference for the optimal control problem. The section is sampled with $200 \mathrm{~Hz}$. Two different LVAD scenarios are evaluated. In the first scenario the scaled in-vivo LVAD flow is used as reference, while in the second one a step from $0 \mathrm{~L} \mathrm{~min}^{-1}$ to $8 \mathrm{~L} \mathrm{~min}^{-1}$ is applied. The pressure reference is the same for both scenarios. Figure 2 shows the reference for both scenarios and the original unscaled invivo trajectories.

\subsection{Results of the Optimal Control Problem}

For each pressure trajectory the mean error is calculated. Figure 3a shows the mean error results of the first scenario for one up to four parallel pumps with the scaled in-vivo data as reference. Less amount of air in the system reduces the mean error significant. The error is further reduced if multiple parallel pumps are used. To reach a mean error of $0.01 \mathrm{mmHg}$ a maximum of around $6 \mathrm{~mL}, 32 \mathrm{~mL}, 53 \mathrm{~mL}$ and $68 \mathrm{~mL}$ unstressed air are allowed to be in the cylinder for 1,2, 3 and 4 parallel pumps. Figure $3 \mathrm{c}$ shows the resulting pressure trajectory for two parallel pumps and $5 \mathrm{~mL}, 30 \mathrm{~mL}, 50 \mathrm{~mL}$ and $100 \mathrm{~mL}$ unstressed air. As the air in the cylinder increases, the pressure trajectories over- and undershoot the reference. The difference in the pressure trajectories of $5 \mathrm{~mL}$ and $30 \mathrm{~mL}$ of unstressed air is nearly impossible to detect as both follow the reference very good. However the mean error for $5 \mathrm{~mL}$ is approximately 10 times lower $(0.00056 \mathrm{mmHg}$ and $0.0058 \mathrm{mmHg}$ ).

Figure $3 \mathrm{~b}$ shows the mean error result of the second scenario. The results show that the step in LVAD flow cannot be compensated by the gear pumps. When there is very few air in the chamber the step has a larger impact on the pressure trajectory, which results in a higher mean error. An increasing amount of air dampens the step input, but results in overall worse pressure trajectories. The minimum mean error is obtained. The position of the minimum slides slightly to higher air volume with more parallel pumps. Figure $3 \mathrm{c}$ shows the resulting pressure trajectories for two parallel pumps and $5 \mathrm{~mL}$,
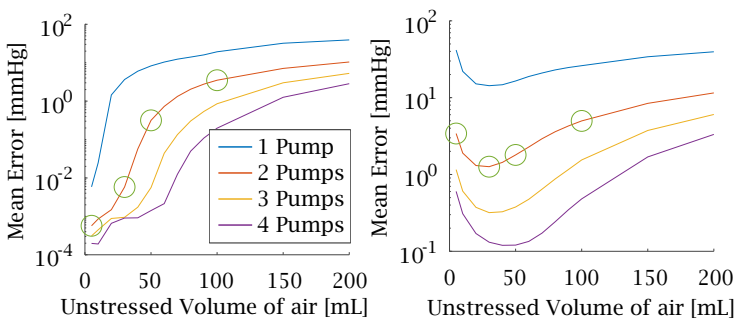

(a) Mean error of the first sce-

(b) Mean error of the second nario.

scenario.

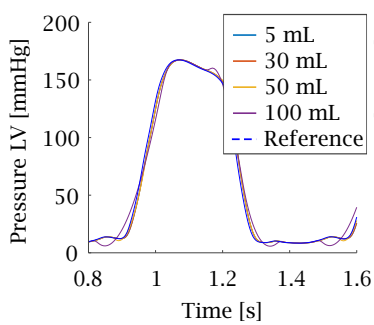

(c) Pressure tracking for two parallel pumps of the first scenario.

Fig. 3: The resulting mean errors and tracking performances for both scenarios of the optimal control problem and a wide range of design parameters are shown.

$30 \mathrm{~mL}, 50 \mathrm{~mL}$ and $100 \mathrm{~mL}$ unstressed air. For the case of $5 \mathrm{~mL}$ of unstressed air the pressure peak at the LVAD step is by far the largest. $30 \mathrm{~mL}, 50 \mathrm{~mL}$ and $100 \mathrm{~mL}$ of unstressed air lead to nearly identical overshoots but with improved reference tracking as the air volume decreases.

Regarding both scenarios, at least two parallel pumps are required to follow the given pressure trajectories. The constant amount of unstressed air should be in a range of $30 \mathrm{~mL}$ to $50 \mathrm{~mL}$ with a slightly better pressure tracking for the chamber with $30 \mathrm{~mL}$ and a slightly better damping for $50 \mathrm{~mL}$ of unstressed air.

\section{Controller Simulation}

The two pressure cylinders are controlled with a cascading controller structure including LVAD feed-forward control. A PID pressure controller delivers the reference values for a subordinate PI flow controller. The controllers are used in a discrete time parallel set-up with a sample rate of $1000 \mathrm{~Hz}$. The PID pressure controller gains are chosen to (bar to $\mathrm{L} \mathrm{min}^{-1}$ ): $P=300, I=8$ and $D=2$. The PI flow controller gains are chosen to ( $\mathrm{L} \mathrm{min}^{-1}$ to $\left.\mathrm{V}\right): P=150$ and $I=6$.

The controller performance is evaluated in Mathworks Matlab Simulink. The pressure and LVAD references are ob- 

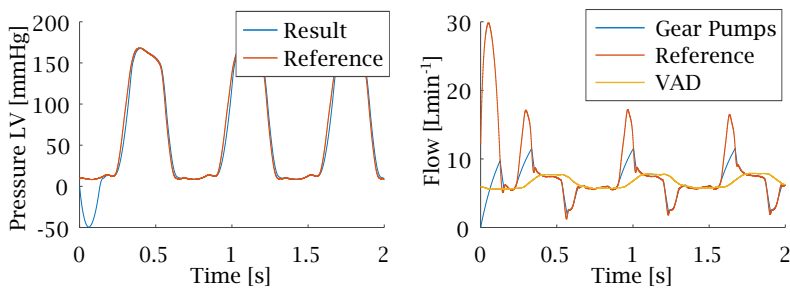

Fig. 4: On the left side the tracking performance of the pressure controller is provided. The right side shows the subordinate flow tracking performance of two parallel gear pumps and the LVAD flow.

tained from the first scenario of the optimal control problem. A set up with two parallel pumps and the identified dynamic parameters is used. $40 \mathrm{~mL}$ of unstressed air are chosen as a good trade off between performance and damping.

Figure 4 shows the results for both controllers. The pressure controller shows good tracking performance after the transients decayed. During the first $0.25 \mathrm{~s}$ the pressure trajectory is not able to follow the reference, as the gear pumps outputs $0 \mathrm{~L} \mathrm{~min}^{-1}$, while the LVAD flow is $6 \mathrm{~L} \mathrm{~min}^{-1}$. If high dynamic positive pressure changes occur in the reference, the system lags slightly behind as the outgoing LVAD flow has to be compensated. At negative pressure changes the problem does not appear, because the LVAD flow decreases the fluid level and the negative pressure change is supported. The same characteristic can be viewed in the subordinate flow controller. At positive pressure changes the reference cannot be followed, due to the limitation of the gear pumps dynamic and voltage. Nevertheless, the overall performance is sufficiently good for the development process of LVAD as long as the LVAD dynamics is not higher than the combined gear pumps dynamics.

\section{Conclusion}

The results demonstrate that the concept works in principle for the left ventricular pressure cylinder. The optimal control problem delivers a suitable range for the amount of air in the cylinder in combination with a number of parallel identified gear pumps. Two parallel pumps and $40 \mathrm{~mL}$ of unstressed air are used in the controller simulation. The results show good tracking performance for a scaled left ventricle in-vivo trajectory as long as the LVAD is not used in a high dynamic range. To validate these findings, further investigations with the MCL under realistic test conditions are required.

\section{Author Statement}

Research funding: This paper was developed during the "LastVAD" project funded by "EFRE", the European Union and the state North-Rhine Westphalia. Conflict of interest: Authors state no conflict of interest. Ethical approval: The research related to animals use complied with all the relevant national regulations and institutional policies for the care and use of animals.

\section{References}

[1] Ferrari G, Kozarski M, et al. (2005). Development of Hybrid (Numerical-Physical) Models of the Cardiovascular System: Numerical-Electircal and Numerical Hydraulic Applications. Biocybernetics and Biomedical Engineering, 25(4), 3-15

[2] Ochsner G, Amacher R, Amstutz A, Plass A, Schmid Daners $M$, Tevaearai Stahel $H$, et al. (2012). A Novel Interface for Hybrid Mock Circulations to Evaluate Ventricular Assist Devices. IEEE transactions on bio-medical engineering. 60 . 10.1109/TBME.2012.2230000.

[3] Telyshev D, Pugovkin A, Selishchev S, Ruschen D, Leonhardt S. (2018). Hybrid mock circulatory loop for training and study purposes. 29-32. 10.1109/USBEREIT.2018.8384542.

[4] Nestler F, Bradley A, Wilson S, L. Timms D, Howard Frazier O, E. Cohn, W. (2014). A Hybrid Mock Circulation Loop for a Total Artificial Heart. Artificial Organs. 38. 10.1111/aor.12380.

[5] Karabegovic A, Hinteregger M, Janeczek C, Reichenfelser W, Soragnese V, Mohl W, Gföhler M. (2014). A systemic mock circulation for in-vitro testing of a pneumatically operated left ventricular assist device. IFAC Proceedings Volumes (IFAC-PapersOnline). 19. 8409-8414.

[6] Gwak KW, Paden B, Noh M, Antaki J. (2006). Fluidic operational amplifier for mock circulatory systems. Control Systems Technology, IEEE Transactions on. 14. 602 - 612. 10.1109/TCST.2006.876624.

[7] Heinke, S. Hard- und Software-Simulation des herzinsuffizienten Kreislaufs zur Entwicklung von Herzunterstützungssystemen. In: Aachener Beiträge zur Medizintechnik: Band 20. Düren: Shaker Verlag; 2014.

[8] Joshi B, Shrestha R, Chaudhary R. (2014). Modeling, Simulation and Implementation of Brushed DC Motor Speed Control Using Optical Incremental Encoder Feedback.

[9] Andersson J, Gillis J, Horn G, B. Rawlings J, Diehl M. (2018). CasADi: a software framework for nonlinear optimization and optimal control. Mathematical Programming Computation. 11. 10.1007/s12532-018-0139-4.

[10] MARCO Data Sheet of UP2-P gear pump. Date accessed: 03.06.2019, https://items.marco.it/en/up2-p-ptfe-gear-pump10-I-min.html

[11] Gesenhues, J. (Publication Pending). Object-Oriented Modeling Based Characterization, Monitoring and Control of the Technically Assisted Cardiovascular System. Dissertation at RWTH Aachen University.

[12] Wächter, A, Biegler, L(2009). IPOPT—an Interior Point OPTimizer. Available from: https://projects.coin-or.org/lpopt. 